\title{
POSMODERNIDAD, DISCURSO Y FRAGMENTO. UNA HIPÓTESIS SOBRE EL DESTINO DEL LIBRO EN EL UNIVERSO DIGITAL
}

\author{
Vicente Serrano Marín \\ Universidad Austral de Chile \\ vicente.serrano@uach.cl
}

La emergencia del universo digital constituye el último gran acontecimiento tecnológico y cultural de Occidente. Se trata de una nueva revolución en una modernidad marcada por el signo de lo revolucionario según la clásica definición de Marx, cuando en el Manifiesto comunista describía el capitalismo al afirmar:

Los continuos cambios en la producción, el incesante sacudimiento de todas las relaciones sociales, la eterna incertidumbre y agitación, destacan la época burguesa entre todas las anteriores. Quedan rotas todas las relaciones fijas y arraigadas, consecuencia de creencias e ideas venerables, mientras las recién establecidas caducan antes de osificarse. Todo lo representativo y permanente se evapora, todo lo sacro es profanado y el hombre se ve al fin obligado a contemplar sin ilusiones su posición en la vida y en las relaciones recíprocas (11).

Hace ya varias décadas, antes incluso de que emergiera lo que hoy conocemos como revolución digital, Marshall Berman había escrito un hermoso libro que toma su título de ese texto de Marx (Berman 2). El texto, dedicado al análisis de la modernidad, fue publicado originalmente en el año 1982, es decir, en las mismas fechas en las que el debate en torno a la llamada posmodernidad parecía estar alcanzando su punto más elevado. Tres años después publicó Habermas su crítica a los que entonces llamaba jóvenes conservadores y a la que dio por título El discurso filosófico de la modernidad. En el prefacio del texto señalaba lo siguiente:

"La Modernidad: un proyecto inacabado" fue el título de un discurso que pronuncié en 1980 al recibir el premio Adorno. Este tema controvertido y rico en facetas no me ha dejado desde entonces. Sus aspectos filosóficos han penetrado más profundamente en la conciencia pública al traducirse las obras de los neoestructuralistas franceses, y también se ha generalizado el término de batalla "postmodernidad" a raíz de una publicación de F. Lyotard (9). 
A su vez, en efecto, la referencia a Lyotard señalaba la obra (Lyotard, La condición postmoderna) que a fines de los años 70 dio carta de naturaleza en el debate filosófico a un término por lo demás antiguo y que, procedente de la estética, se había consolidado con anterioridad en el ámbito de la arquitectura. Si señalamos estas fechas es para recordar que la cuestión de la posmodernidad coincide con los primeros pasos de lo que por entonces se empezaba a llamar la sociedad de la información y que con el tiempo acabaría por convertirse en la revolución digital en la que hoy vivimos (Salvat y Serrano, La revolución digital y la sociedad de la información 23-25). Las transformaciones de lo que Lyotard mismo llamaba informatización de la sociedad (21), han dado lugar a esa revolución que constituye hoy el horizonte ineludible al que nos asomamos en el presente. Por el contrario, aunque la noción de posmodernidad goza de una considerable salud más allá de los términos filosóficos en los que fue planteado al final del pasado siglo, lo hace al precio de una ambigüedad y de un carácter difuso y el debate estrictamente filosófico en torno a la misma nos resulta ya lejano. En ello influyó en parte el llamado affaire Sokal, en el que Alan Sokal y Jean Bricmont denunciaban los "abusos intelectuales" de muchos de los autores considerados posmodernos (Sokal y Bricmont, Imposturas intelectuales 30-31), pero también el transcurso del tiempo, que fue mostrando cómo algunos de esos autores no eran tan posmodernos y cómo algunas obras que pretendieron defender de manera abierta la posmodernidad se han ido diluyendo en una considerable irrelevancia. Respecto de lo primero es llamativo el caso de Foucault, a quien Habermas etiquetó en su día de posmoderno y antiilustrado en el texto señalado más arriba (285-310). En 1984, poco antes de su muerte, se desmarcó de una etiqueta a la que consideraba "enigmática" (Foucault, ¿Que es Ilustración? 11) y se pronunció como un defensor de la Ilustración. Sin embargo la cuestión de qué se debe entender por posmodernidad está aún lejos de haber sido resuelta y merece una reflexión detenida, especialmente tras la emergencia del universo digital en el que ya vivimos. Como parte de esa reflexión nuestro objetivo aquí es hacer un acercamiento a la idea misma de lo digital desde las reflexiones en torno a lo posmoderno y a su vez comprender mejor lo que significó la llamada posmodernidad a partir de la información de que hoy disponemos en torno al universo digital. Y ambas cosas precisamente a partir de la dimensión narrativa del saber.

La hipótesis que quisiéramos desarrollar tiene que ver con una interpretación de lo que podemos entender por la noción de gran relato en su relación con lo digital. $\mathrm{O}$ por decirlo de otro modo, el objetivo es ensayar una definición de lo digital a partir del debate posmoderno en torno a la noción de relato y las dimensiones narrativas del saber. Cuando se acude en busca de una definición de lo digital por contraposición a lo analógico es frecuente situar las diferencias en el ámbito técnico referido al tipo de tecnología, en particular a la clase de señal que se da en cada una de las tecnologías. Ese tipo de definición es sin embargo poco aclaratoria de las consecuencias de lo digital para la sociedad y la civilización en su conjunto. La otra opción es acudir directamente a esas consecuencias y hacer entonces una descripción de lo digital desde el punto de vista de los cambios sociales y culturales. Entre esos dos extremos, que, o bien se quedan cortos y son ciegos, o bien son excesivos y vacíos al subsumir y diluir lo digital en otras denominaciones como sociedad de la información, capitalismo tardío, sociedad riesgo o sociedad red, entre otros (Salvat y Serrano 18-19), es posible y necesario ofrecer una 
definición de lo digital, y por tanto también de lo analógico, que atienda a la dimensión lingüística que atraviesa la cultura contemporánea y que ha marcado las ciencias sociales y humanas mediante el llamado giro lingüístico y que al hacerlo dé cuenta tanto de las diferencias técnicas como de las sociales y culturales.

Un primer aspecto a considerar viene determinado por el hecho de que lo que llamamos analógico solo se hace visible a partir de la emergencia de lo digital. En ese sentido, la noción de lo analógico tiene la apariencia de un objeto nuevo. Y sin embargo no lo es, propiamente hablando. Esa apariencia se genera precisamente porque hasta la emergencia de la tecnología digital todo era analógico y, faltando esa capacidad de diferenciarlo por oposición a otra realidad, no resultaba visible. De algún modo lo digital además de su propia presencia ha hecho visible la de aquello a lo que viene a sustituir. Aunque, como señalábamos más arriba, a la hora de definir nuestro objeto no parece suficiente referirse únicamente a los aspectos técnicos que envuelven a cada una de las tecnologías, sí parece necesario partir de ahí para avanzar en la dimensión esencial que buscamos. A este respecto cualquier manual básico de medios digitales nos explica los diferentes modos de representar la magnitud matemática que llamamos señal y que hace posible las telecomunicaciones. Su rasgo fundamental es el carácter discontinuo o discreto de la señal, organizada en términos de 1 y 0 a partir de la herramienta que nos ofrece el álgebra de Boole. Frente a ella, la señal analógica representa las magnitudes en términos de continuidad y esa continuidad es la que le da el nombre de analógica. Ambas son abstracciones, pero la representación digital no requiere ya de la proporcionalidad en un continuo, como ocurre por ejemplo al medir la velocidad mediante una aguja o la temperatura en un termómetro mediante el mercurio (Tocci, Ronald J. y Wider, Neil S. Sistemas digitales, principios y aplicaciones 4). Por el contrario, en la representación digital las cantidades no se representan proporcionalmente, sino que en ella se opera únicamente mediante esos símbolos llamados dígitos. Esto se aprecia bien intuitivamente en la diferencia entre el reloj analógico y el digital. En el reloj analógico la hora cambia de forma continua tal como apreciamos en nuestra experiencia del tiempo o del movimiento de las agujas en la esfera de un reloj analógico, mientras que en el reloj digital ese cambio se produce cada minuto, en su caso en cada segundo, es decir, a saltos representados mediante dígitos.

Como se puede apreciar, lo analógico y lo digital tienen en común ser modos de representar matemáticamente la experiencia. Sin embargo, la matematización de la experiencia es algo poco novedoso, al menos en el mundo moderno, y más bien constituye la definición básica de lo que llamamos ciencia desde que Galileo propuso el ideal de una ciencia en la que el libro de la naturaleza está escrito en caracteres matemáticos. ¿Dónde reside entonces la novedad de lo digital? ¿en qué se diferencia la electrónica analógica de la digital si ambas están basadas en criterios matemáticos? Más aún ¿no supone cualquier matematización de la experiencia de hecho el establecimiento de valores discretos y discontinuos?

Hay una respuesta sencilla a esas preguntas y tiene que ver con la representación. En efecto, es en el ámbito de la representación en el que la señal analógica lo es. Un sistema analógico contiene dispositivos que manipulan cantidades físicas que a su vez son representadas en forma analógica. Lo analógico es una representación o se mueve en 
el ámbito de la representación. Con frecuencia se afirma que las cosas que se pueden medir cuantitativamente se presentan en la naturaleza en forma analógica, pero se obvia explicar qué se entiende entonces por naturaleza, o, más bien, qué se entiende por la expresión aparecen en la naturaleza de forma analógica. Es evidente que los números que miden las cosas en la naturaleza no son analógicos y son más bien discontinuos por definición, pues el término mismo procede del modo de representar un solo guarismo al que se llama dígito. Pero lo que llamamos analógico no remite a esa abstracción en forma numérica con la que opera la ciencia, sino más bien con la entidad sobre la cual recae la medida y que fuera de esa medida resulta incognoscible para la ciencia moderna. Lo analógico es una nueva representación que hacemos de esa entidad una vez medida por la ciencia. Esta idea es la que había expresado Immanuel Kant en su esfuerzo por sintetizar las características y el modo de proceder de la ciencia moderna. En efecto, en la Crítica de la Razón Pura, Kant dejó claro que no tenemos acceso a lo que él llamó cosa en sí para referirse a esa entidad a medir $(73-74,87)$, sino que más bien construimos mediante las formas a priori de la sensibilidad, en términos de espacio y tiempo, las cuales introducen ya las magnitudes que hacen posible la experiencia. A esa entidad sobre la que recae la magnitud la declaró incognoscible. A su vez lo que llamamos naturaleza se reduce a partir de ese momento a un "compendio de fenómenos" (140) como resultado de esa matematización inicial. Nuestro conocimiento de lo que llamamos naturaleza no es tanto de esta entidad en cuanto lo que es en sí, sino de los fenómenos discontinuos que vamos construyendo al matematizarla, de manera que "somos, pues, nosotros mismos los que introducimos el orden y regularidad de los fenómenos que llamamos naturaleza" (148). Y en ese sentido esa unidad de la que depende lo que acostumbramos a llamar naturaleza al hablar de lo analógico no es sino una ficción reguladora (549-550). Dicho de forma breve, no cabe una medida, ni por tanto experiencia, del conjunto y de la totalidad de la naturaleza y dado que no hay conocimiento allí donde no hay experiencia esa idea de la naturaleza como totalidad es únicamente una ficción. Es decir, constituye una nueva representación que como tal no es conocimiento y en el mejor de los casos tiene únicamente un valor regulativo. Se trata de un tipo de representación que se sitúa próxima al arte y de hecho solo así se accede a esa naturaleza entendida como totalidad, a la naturaleza en su conjunto como un todo continuo. Kant la va a reconsiderar en la tercera Crítica, en el mismo lugar donde trata el arte y lo bello. Desde el punto de vista de su consistencia discursiva la naturaleza que mentamos en la expresión "las cosas que se pueden medir cuantitativamente se presentan en la naturaleza en forma analógica" es idéntica a cualquier representación artística por contraposición a lo científico, si bien es cierto que en este contexto posee una función que podemos llamar epistemológica o cognoscitiva, que es lo que Kant llamó idea regulativa, es decir, una representación ficticia que acompaña al conjunto de los fenómenos obtenidos por la ciencia para darles una continuidad que estos no poseen, para darles lo que podríamos llamar, ahora ya sí, un carácter analógico.

Cuando Lyotard a fines de los años 70 ofrecía una descripción de la posmodernidad como pérdida de la fe en los grandes relatos, lo hacía a partir de una distinción en el seno del saber entre lo que llamaba el saber científico y lo que denominaba el saber narrativo (Lyotard, 43-50). El primero, que tiene que ver con el surgimiento de lo que llamamos 
ciencia en sentido moderno, se caracteriza fundamentalmente por excluir del campo del saber todas las dimensiones no denotativas, es decir, por excluir los enunciados de preferencia o de valoración, por excluir, en definitiva, todo aquello no susceptible de matematización, como la ética o la estética. En cambio el saber narrativo, que habría sido la forma del saber previo a la modernidad y a la ciencia, habría contenido reunidas todas esas dimensiones, la denotativa susceptible de verdad y falsedad, y las dimensiones valorativas y éticas que atribuimos a la ética y a la estética. A partir de esta distinción básica la tesis de Lyotard se completaba con una reflexión acerca de cómo la modernidad en sus primeros pasos necesitó recubrir a la ciencia mediante un relato que recobrara y contuviera las dimensiones perdidas del saber narrativo que son desechadas por la ciencia (63). La noción de grandes relatos tenía que ver con ese esfuerzo y esa necesidad narrativa que debía acompañar a la ciencia (58-60). El referente de la modernidad, sobre la que recaía el discurso posmoderno para señalar su fin, no era tanto la ciencia moderna, sino más bien el de los relatos que la acompañaron. Lo que se declaraba en su definición de la posmodernidad era el carácter obsoleto e innecesario de esa dimensión narrativa que durante los siglos modernos había acompañado a la ciencia (73).

De ser cierta esta tesis tendríamos una aproximación a lo analógico que, lejos de limitarse a los aspectos técnicos circunscritos a la electrónica, permite igualmente dar cuenta de muchos de los fenómenos culturales y de las consecuencias sociales de lo digital. Sin embargo, nuestra indagación partía de considerar esa posible definición de lo analógico como dependiente a su vez de la emergencia de una nueva tecnología, de una nueva realidad que es la que había hecho visible la existencia de lo analógico. Con la explicación que hemos ofrecido hasta aquí tenemos una comprensión inicial: estaríamos ante una textura narrativa que se considera innecesaria. ¿Pero qué ocurre con la nueva tecnología? ¿qué lugar ocupa en este contexto? ¿reemplaza sin más al relato moderno? A este respecto conviene no olvidar que cuando hablamos de lo digital nos situamos, como ocurría respecto de lo analógico, en el ámbito de la representación. Recordemos igualmente que lo digital es, además de una tecnología, un lenguaje, y como tal es también y por definición, discurso. Por tanto desde el punto de vista estrictamente tecnológico no hay duda de que el lenguaje digital ha desplazado y sustituido al analógico, puesto que constituye un nuevo modo de representar las señales y ese ha sido nuestro punto de partida. Pero la pregunta es si lo es también desde el punto de vista de lo que podríamos llamar la dimensión narrativa del saber, es decir, de aquellas otras dimensiones contenidas en el saber analógico. O por decirlo de otra manera y dotando ya de contenido a lo uno y lo otro: ¿constituye el mundo digital un relato alternativo al que hemos construido y comprendido como analógico?

Una estrategia adecuada para responder a esa cuestión sería indagar las características propias de ese nuevo modelo de representar, lo que nos exige de nuevo volver a la contrastación con el anterior, es decir, con el que hemos descrito y descubierto como modo analógico. Sin embargo debemos evitar en este sentido identificar ninguno de ellos con la noción de realidad u otras semejantes, puesto que el territorio común de ambos es el de la representación. Por tanto no sería válida una aproximación en la que insinuáramos que el universo digital no es real y sustituye por así decir la realidad que estaría encerrada en el analógico. Lo propio del relato analógico no descansaba en su 
condición de realidad sino en el hecho de ofrecer una totalidad en la que subsumir los elementos discontinuos y situar cualquier acontecimiento en el interior del mismo. De hecho tanto el término relato como el término analógico remiten a la idea de unidad o más bien de unificación.

En efecto, por relato entendemos aquella trama discursiva que es capaz de relacionar distintos objetos en una unidad y la relación a su vez no es más que el vínculo que reúne a partir del juego entre el prefijo latino que introduce la idea de reiteración o regreso y que precede al verbo fero. De la combinación de ambos se obtiene la idea de una acción de traer de nuevo aquello que se encuentra separado para reunirlo. Un relato es fundamentalmente una unidad discursiva capaz de anudar múltiples acontecimientos, una totalidad. Por su parte la noción de lo analógico remite a la combinación de un prefijo relativo a la preposiciones castellanas por o sobre y combinadas con el término logos hace referencia a la noción discursiva que está por o sobre dos cosas diferentes para reunirlas en el discurso a partir de ese elemento común.

No puede ser casual en este sentido que el relato analógico más antiguo que poseemos históricamente en la cultura occidental acaba por condensarse en torno al término ser, puesto que el ser es el vínculo o la cópula en el juicio, es decir, en el ámbito discursivo que es el propio de la representación y en el que situamos tanto lo analógico como lo digital. El saber propio de la metafísica premoderna era un saber narrativo, un saber cualitativo mediante el que se reúne la totalidad de las diferencias a partir de una noción común no mensurable y por tanto no susceptible de la discontinuidad de las cuantificaciones. En su momento maduro el concepto de ser que articula la metafísica de Aristóteles era a su vez una reelaboración abstracta a partir de las primeras indagaciones que elaboraron los presocráticos presentadas habitualmente en forma de poema bajo el título En torno a la naturaleza. Más allá de su condición abstracta y de la áspera presentación que de las nociones metafísicas hizo Aristóteles, el ser dependía de un cuadro en torno al mundo, ordenado en torno a múltiples esferas y que estuvo vigente como su sustrato hasta la emergencia de la ciencia moderna, es decir, de aquel modo de saber que rompe con lo narrativo y que al hacerlo exige construir un relato que acompañe a la ciencia, al que hemos llamado grandes relatos y del que hemos derivado la noción de analógico como aquella representación que la acompaña.

Al surgir la ciencia moderna, la noción de ser como entidad cualitativa fue perdiendo la centralidad que había poseído. Ya en Kant pasó a convertirse en la posición absoluta de una cosa o el nexo en los enunciados (Crítica de la razón pura 504). Su uso se prolongó mediante el relato moderno en distintas manifestaciones metafísicas que intentaron sustituirlo o reelaborarlo y su última expresión fue tal vez el esfuerzo heideggeriano, que sin embargo reconocía ya esa textura narrativa al situarlo habitando en el lenguaje (Heidegger, Carta sobre el humanismo 11). Pero en la posmodernidad el lenguaje se desentiende definitivamente del ser y su prolongación en forma de relato analógico llega ahí a su fin. En su lugar se instala el lenguaje sin un referente al que representar en forma de relato y que entonces se representa a sí mismo en un reenvío interminable. Esa es el signo común de todos los discursos de finales del siglo XX en el ámbito de las distintas tradiciones marcadas por el llamado giro lingüístico. Nuestra hipótesis es que esa disolución del último resto del ser en el lenguaje habría sido algo así como un 
anticipo todavía metafísico, o al menos especulativo, del acontecimiento de lo digital que se está gestando en los mismos años en el que teóricos procedentes de distintas disciplinas debaten en el seno de la primacía de lo lingüístico que ha marcado el fin del siglo XX.

La fragmentación es el fenómeno que mejor puede confirmar esa hipótesis, en la medida en que constituye un rasgo común del discurso posmoderno y del lenguaje digital y en la que esa pérdida del último resto narrativo del lenguaje sería el correlato especulativo del carácter discreto de lo digital. Sobre ella hay un considerable consenso y creemos que esa fragmentación podría constituir una especie de corroboración que vendría a confirmar la hipótesis sobre el paralelismo que hemos establecido entre posmodernidad y digitalización de la sociedad. Así las cosas, de modo provisional podríamos responder ya a la pregunta acerca de la textura narrativa de lo digital y afirmar que, en efecto, estamos ante un discurso que ha dejado de ser narrativo, si por narrativo entendemos la idea misma de un vínculo capaz de unificar los acontecimientos.

Ahora bien, desde el punto de vista de la historia de la literatura y de la estética, el fragmento en sí mismo está lejos de ser ajeno a lo narrativo. Hay dos momentos en la cultura occidental moderna en el que el fragmento ocupa un lugar preeminente, lugar que no ha abandonado desde entonces. El primero de esos momentos es el Romanticismo inicial, cuya figura más destacada fue Friedrich Schlegel. La fuente principal al respecto se halla en los Fragmentos del Ateneo, una obra que no solo incluye el término fragmento en su propio título, sino que posee en sí misma una estructura fragmentaria. En el fragmento 116, en el que se formula el ideal discursivo de la escuela entendido como poesía universal progresiva, ésta aparece configurada como "un espejo de la totalidad del mundo circundante" (69). El sentido del fragmento romántico lejos de ser la ausencia de relato es la forma en que una primera reflexión sobre la modernidad asume la posibilidad de mantener una textura narrativa en un contexto en el que el dominio de la ciencia y de lo cuantitativo había expresado esa fuerza desintegradora a la que Kant, como vimos, había dado respuesta en términos epistemológicos y los románticos en términos estéticos. El fragmento romántico es en realidad un recurso estético frente a la fragmentación moderna, pero lo es en la medida en que esa noción de totalidad sigue operando como trasfondo, aunque se exprese de modo fragmentario.

El otro momento en el que el fragmento ha tenido un protagonismo decisivo fue la emergencia de la Enciclopedia como discurso fragmentario. En ella, en efecto, el fragmento puede leerse desvinculado de la totalidad de la obra (Chartier, ¿Morirá el libro? Entrevista al doctor Roger Chartier, 190). Sin embargo, más allá de esa ausencia de la dimensión narrativa que define el texto enciclopédico, no puede olvidarse que constituyó un esfuerzo por reunir el conjunto del saber -no solo de las ciencias físicas y matemáticas- en una unidad y bajo un presupuesto común que era una determinada imagen del mundo y que se pretendía a sí misma como "un cuadro general de los esfuerzos del espíritu humano en todos los órdenes y durante los siglos". Esa imagen del mundo estaba dominada por la idea de progreso, por el optimismo ilustrado en torno a la razón, por la creencia compartida acerca de la naturaleza y se expresaba en forma figurada mediante una representación de lo que se llamaba el árbol humano del conocimiento articulado en torno a la imaginación, la razón y la memoria. Es decir, la misma que será expresada en breve por la metafísica moderna que se desplegará en la filosofía de la historia y de 
la que va a nacer el gran relato moderno en el sentido estricto que le da Lyotard en $L a$ condición posmoderna.

La fragmentación digital, que según nuestra hipótesis fue anticipada por la especulación posmoderna, se mueve en un ámbito diferente. No contiene implícito un universo bajo la forma fragmentaria de cada artículo, ni es la metáfora de un relato, que como tal remite a una totalidad, como ocurre en el Romanticismo, sino que se instala en una fragmentación radical y sin retorno. El problema lo ha explicitado Roger Chartier con claridad y precisión:

La práctica de seleccionar pasajes, aun en el libro impreso, remite a la totalidad de la obra. El fragmento está dentro de esa totalidad, inclusive si el lector no ha leído todas las páginas, porque hay elementos paratextuales que indican algo sobre el conjunto de la obra. La fragmentación de la lectura frente a la pantalla no remite a la totalidad de la obra. Hoy se utilizan los extractos sin ninguna relación con la totalidad en obras que fueron concebidas como una totalidad. Esta es una diferencia profunda y radical [.... Mientras que alguien puede leer tres páginas de Madame Bovary en e-book y destacar esas páginas, que adquieren una identidad y una vida singular, pero que no remiten más al proyecto estético de Flaubert. Es una diferencia importante que hay que considerar, la nueva relación entre el fragmento y la totalidad, que debería generar dispositivos que permitan reconstruir algo de esa totalidad en el soporte electrónico [...] El nuevo mundo textual, en un futuro que nos desborda, puede ser un futuro de fragmentos textuales (Entrevista a Roger Chartier. http://www.ddooss.org/articulos/ textos/Roger_Chartier.htm).

Cualquier recorrido por el universo digital corrobora esa apreciación. Estamos ante un universo en movimiento que no parece remitir a un todo capaz de integrar en él cada uno de los fragmentos a los que accedemos a través de la pantalla. La ya vieja idea del hipertexto, por lo demás tan estrechamente análoga al posmoderno reenvío derrideano (Derrida, De la Gramatologie 90), se ha extendido hasta articular un nuevo tejido que no puede remitir ya al libro de libros que era la Biblia. Tampoco al relato que acompañaba a la Enciclopedia. Más allá de ese carácter formalmente fragmentario que hemos mencionado, la Enciclopedia remitía igualmente a una totalidad comparable a la Biblia en la época de la Ilustración y de la ciencia moderna y a partir de un tejido narrativo que alimentó los dos grandes relatos modernos identificados por Lyotard en La Condición posmoderna: el de la vida del espíritu capaz de autorrealizarse mediante la formación entendida como Bildung, al que llama relato especulativo, y el de la emancipación de los hombres mediante los procesos revolucionarios, al que llama relato de la emancipación (73).

La preocupación expresada de modo tan preciso por Chartier va entonces más allá de la cuestión formal. Parece afectar a una instancia en la que nuestra hipótesis acerca del paralelismo entre lo digital y la especulación anticipadora de la posmodernidad muestra una considerable capacidad heurística. Desde esa hipótesis, el problema del fragmento y su relación con la totalidad no descansaría tanto en los formatos como en el propio discurso, no en la forma del relato como en el hecho de que el relato se disuelve. La transformación de los modos de escritura y lectura que estamos viviendo como consecuencia de la nueva tecnología constituye así el punto de encuentro entre las dimensiones tecnológicas y culturales en sentido amplio, a las que nos referíamos 
al comienzo de estas páginas en nuestra búsqueda de una definición de lo digital. En la tensión que el libro vive como forma confluye tanto una concepción de la cultura de Occidente, a la que ha acompañado desde que se constituye como tal, como esa expresión técnica acerca del modo en que leemos y escribimos. Aunque en su etimología tiene que ver con una unidad material, articulada en torno al papiro egipcio en el caso del término griego, o a la corteza del árbol en el término latino (Barbier, Historia del libro 9-10), muchos siglos antes de la imprenta y también antes de que la UNESCO diera la escueta definición del libro, es obvio que el libro posee además un sentido que trasciende esa materialidad y abraza en ella las formas sociales políticas, culturales y económicas de cada época (Barbier 11). En la medida en que una definición como esa es a la vez inevitablemente una aproximación a la lectura y a la idea misma de texto y de escritura, el libro sería una adecuada metáfora para identificar lo analógico, precisamente porque en esa doble condición material y espiritual expresa y encierra la idea de totalidad que hemos tratado de expresar a partir de la continuidad, desde ese punto de vista, entre la Biblia y la Enciclopedia ilustrada. Como realidad material el libro expresa, en su forma definida y cerrada, una unidad que es una totalidad en sí misma, y aspira a reflejar a su vez la totalidad del mundo que asociamos a lo analógico, en la que la escritura, la comunicación y la memoria encontraron durante siglos un cauce formal válido.

La ruptura que supone la emergencia de lo digital y el modo como afecta al libro sería la manifestación más visible de un nuevo tejido discursivo ajeno ya a la dimensión narrativa. En este sentido, lo que está en cuestión no es tanto que el viejo libro desaparezca como objeto material, lo que no parece probable que ocurra nunca, ni tampoco el uso que el libro digital puede tener. Lo que está en cuestión es el tejido discursivo en el que se articula nuestra interacción con el mundo y si su sustrato último depende de la fragmentación propia de la vida en las pantallas y que hasta hace poco tiempo descansaba en los libros. O, dicho de otra manera, la pregunta es si el modo que tendencialmente domina nuestra forma de representar y de representarnos no está ya configurado por el libro como expresión analógica y metáfora del mundo, sino por la forma discursiva digital, con respecto a la cual el libro entendido en sentido estricto seguirá conviviendo, pero subsumido como una expresión entre otras para ser un fragmento entre fragmentos. Es ahí donde se cruza el problema de la fragmentación y la cuestión de la textura narrativa propia de lo digital. El problema no sería ya entonces determinar cómo se comportan los libros respecto de lo digital o lo digital respecto de los libros, sino más bien en qué medida ese nuevo formato dominante que es la red, articula discursivamente nuestras vidas y los modos que tenemos de representarlas, al margen del modelo del libro que ha dominado en Occidente. Se trataría de un tránsito que va más allá de la superación de la galaxia Gutenberg, si por libro debemos entender aquí una estructura que va más allá de la invención de la imprenta y que abarca todas las formas de escritura anteriores a la era digital, como precisa Chartier al recordarnos que "la imprenta no altera las modalidades de la relación con lo escrito" (Libros, lecturas y lectores 23).

Baudrillard iniciaba su obra Cultura y Simulacro evocando un relato de Borges donde el mapa se superpone a la realidad, superposición a la que denominaba lo hiperreal (5). Pero si asumimos, como hemos hecho a lo largo de este trabajo, que no es sobre lo real sobre lo que opera la diferencia entre analógico y digital, pues ambos no son sino 
lenguajes y texturas discursivas, el problema no reside en el referente, sino más bien en el sentido, esa dimensión del discurso que redescubre Frege y está más allá del referente. Durante siglos lo analógico, en sus distintas versiones, ha sido capaz de ofrecer el sentido. Y desde ese punto de vista es otro relato de Borges el que acierta a expresar lo digital de un modo más preciso. En el Aleph ya no es el libro sino una piedra, apenas unos centímetros más pequeña que la pantalla de un teléfono celular, la que permite asomarse a esa proliferación de sentidos, cuyo "problema central es irresoluble: la enumeración, siquiera parcial, de un conjunto infinito".

\section{BIBLIOGRAFÍA}

Barbier, Frederic. Historia del libro. Madrid: Alianza, 2005.

Baudrillard, Jean. Cultura y simulacro. Barcelona, Kairós, 1978.

Berman, Marshall. Todo lo sólido se disuelve en el aire. Madrid: Siglo XXI, 1988

Chartier, Roger. Libros, lecturas y lectores de la Edad Moderna. Madrid: Alianza, 1993.

Cultura escrita, literatura e historia. México: FCE, 1994

189-194.

¿Morirá el libro? Entrevista al doctor Roger Chartier. Revista Historia (2012):

Entrevista a Roger Chartier. Por Silvina Friera. 13 de junio de 2010. http://www. ddooss.org/articulos/textos/Roger_Chartier.htm

Las revoluciones de la escritura. Diálogos e intervenciones. Barcelona: Gedisa, 2009.

Derrida, Jacques. De la Gramatologie. Paris: Minuit, 1967.

Foucault, Michel. ¿Que es Ilustración? Daimon 7 (1993): 5-18.

Habermas, Jürgen. El discurso filosófico de la modernidad. Madrid: Taurus, 1985.

Heidegger, Martin. Carta sobre el humanismo. Madrid: Alianza, 2000.

Kant, Immanuel. Crítica de la razón pura. Madrid: Alfaguara, 1997.

Lyotard, J-F. La condición posmoderna. Un informe sobre el saber. Madrid: Cátedra, 1987.

Marx, Carl y Friedrich Engels. El manifiesto comunista. Santiago de Chile: Editorial Universitaria, 1970.

Salvat, Guiomar y Vicente Serrano. La revolución digital y la sociedad de la información. Zamora/Sevilla: Comunicación Social, 2011.

Schlegel, Friedrich. Fragmentos del Ateneo. En Onetto, Breno y Gonzalo Portales. Editores. Poéticas de la infinitud, pp. 25-254. Santiago: Palinodia, 2005.

Sokal, Alan y Bricmont, Jean. Imposturas intelectuales. Barcelona: Paidós, 1999.

Tocci, Ronald J. y Neil S. Wider. Sistemas digitales, principios y aplicaciones. México: Pearson educación, 2003. 